\title{
ON THE CLASSIFICATION OF GEOMETRIC FAMILIES OF FOUR-DIMENSIONAL GALOIS REPRESENTATIONS
}

\author{
Luis Dieulefait AND NÚRIA Vila
}

\begin{abstract}
We give a classification theorem for certain four-dimensional families of geometric $\lambda$-adic Galois representations attached to a pure motive. More precisely, we consider families attached to the cohomology of a smooth projective variety defined over $\mathbb{Q}$ with coefficients in a quadratic imaginary field, non-selfdual and with four different Hodge-Tate weights. We prove that the image is as large as possible for almost every $\lambda$ provided that the family is irreducible and not induced from a family of smaller dimension. If we restrict to semistable families an even simpler classification is given. A version of the main result is given for the case where the family is attached to an automorphic form.
\end{abstract}

\section{Introduction}

In this paper we study compatible families of four-dimensional Galois representations. We obtain a classification theorem for families of geometric Galois representations attached to a smooth projective variety defined over $\mathbb{Q}$, with coefficients in a quadratic imaginary field, non-selfdual and with four different Hodge-Tate weights. In particular we have generically large images for those families of Galois representations which are irreducible and not induced from a family of smaller dimension.

This result improves our results of [6] where generically large image for four-dimensional families of Galois representations are obtained, assuming that certain explicit conditions are satisfied.

The first large image result of this kind was obtained by Serre for the case of elliptic curves without Complex Multiplication (cf. [13]). Serre also obtained a large image result for the four-dimensional Galois representations attached to principally polarized abelian surfaces with trivial endomorphism ring (cf. [14]), in his result he deals only with symplectic representations, while we will work with four-dimensional representations which are not symplectic. Results of generically large images were obtained by the authors in [5] for compatible families of three-dimensional Galois representations.

The Setup: Precise definition of the kind of Galois representations that we are we going to study

Consider a compatible family of four-dimensional Galois representations

$$
\rho_{\lambda}: G_{\mathbb{Q}} \rightarrow \operatorname{GL}\left(4, K_{\lambda}\right),
$$

that appear as subquotient of a family of $\ell$-adic Galois representations attached to the Galois action on some étale cohomology groups $H_{e t}^{n}\left(X_{\overline{\mathbb{Q}}}, \mathbb{Q}_{\ell}\right)$ of a smooth projective

Received by the editors March 7, 2011. Revision received April 28, 2011. 
variety $X$ defined over $\mathbb{Q}$. We say that the family $\left\{\rho_{\lambda}\right\}$ is a geometric four-dimensional compatible family of Galois representations of $G_{\mathbb{Q}}$. We assume that $K$ is a quadratic imaginary field and that the Hodge-Tate numbers are $\{0,1,2,3\}$, with the simplifying assumption that the determinants are just $\chi^{6}$, where $\chi$ is the cyclotomic character. For any prime $\lambda$ in $K$, we will always call $\ell$ the rational prime below it.

Let $N$ denote the product of the bad reduction primes of the variety $X$. As is well-known such a compatible family has a ramification set contained in the set of prime divisors of $N$ and is pure, i.e., the four roots of the characteristic polynomial of the image $\rho_{\lambda}$ (Frob $p$ ), for any unramified place $p$ prime to $\lambda$, have the same absolute value.

From the compacity of $G_{\mathbb{Q}}$ and the continuity of the representations $\rho_{\lambda}$, it follows that we can assume that the images are contained in $\operatorname{GL}\left(4, \mathcal{O}_{\lambda}\right)$, where $\mathcal{O}$ denotes the ring of integers of $K$. This implies that we can consider the residual representations $\bar{\rho}_{\lambda}$ with values in $\operatorname{GL}\left(4, \mathbb{F}_{\lambda}\right)$, obtained by composing $\rho_{\lambda}$ with the naive map "reduction modulo $\lambda . "$

$$
\bar{\rho}_{\lambda}: G_{\mathbb{Q}} \rightarrow \mathrm{GL}\left(4, \mathbb{F}_{\lambda}\right) .
$$

Since this reduction is only well-defined up to semi-simplification, we consider its semi-simplification, which we are going to denote $\bar{\rho}_{\lambda}$ by abuse of notation. Thus, from now on all residual representations will be assumed to be semi-simple.

Observe that from purity it follows that if we denote by $a_{p}$ the trace of the characteristic polynomial of $\rho_{\lambda}\left(\right.$ Frob $p$ ) for any prime $p \nmid \ell N$, it holds that $\left|a_{p}\right| \leq 4 p^{3 / 2}$.

The characteristic polynomials of $\rho_{\lambda}$ (Frob $\left.p\right)(p \nmid \ell N)$ will be denoted

$$
x^{4}-a_{p} x^{3}+b_{p} x^{2}-p^{3} \bar{a}_{p} x+p^{6} .
$$

The particular form of these polynomials and the fact that $b_{p} \in \mathbb{Z}$ follows, as is well known, from purity. We assume that the values $a_{p}$ generate $K$.

Remark. We require that the traces at Frobenius elements generate $K$, an imaginary quadratic field, because in the opposite case, if all traces are real, the representations would be selfdual, thus for all primes $\lambda$ the images would not be "as large as possible" (in fact, the images would be contained in an orthogonal or a symplectic group). Observe that even with the assumption that the traces generate $K$, the particular form of the characteristic polynomials, together with Cebotarev density theorem imply that for any prime $\lambda$ inert in $K$ the image of $\rho_{\lambda}$ will be contained in the unitary group $\mathrm{GU}\left(4, \mathbb{Z}_{\ell}\right)$.

By conductor of the representations $\rho_{\lambda}, \operatorname{cond}\left(\rho_{\lambda}\right)$, we mean the prime-to- $\ell$ part of the Artin conductor. It is known that the conductor of the representations $\rho_{\lambda}$ is bounded independently of $\ell$, because the representations are geometric. This follows from Deligne's corollary to the results of de Jong (cf. [2, Proposition 6.3.2]). We will denote by $c:=$ l.c.m. $\left\{\operatorname{cond}\left(\rho_{\lambda}\right)\right\}_{\lambda}$ this finite uniform bound, and we will call it "conductor of the family of representations."

Let $d$ denotes the the greatest integer such that $d^{2} \mid c$.

Condition (A). There exists a prime $p$ congruent with -1 modulo d, such that none of the roots of $Q_{p}(x)$, the characteristic polynomial of $\wedge^{2}\left(\rho_{\lambda}\right)($ Frob $p)$, is equal to $-p^{3}$. 
Remark. If the conductor $c$ is square-free we set Condition (A) to be the empty condition.

This finishes the description of the geometric families of Galois representations that we are going to consider. The following is the main theorem of this paper, and it applies to them:

Theorem 1.1. Let $\left\{\rho_{\lambda}\right\}$ be a geometric four-dimensional compatible family of Galois representations of $G_{\mathbb{Q}}$ with coefficients generating an imaginary quadratic field $K$, with Hodge-Tate numbers $\{0,1,2,3\}$, with determinant $\chi^{6}$ and satisfying Condition (A). Assume that there exists a good reduction prime $p$ such that $\mathbb{Q}\left(a_{p}^{2}\right)=K$ and $b_{p} \neq 0$.

Then one of the following holds:

(i) There exists $f_{1}$ and $f_{2}$ classical cuspidal modular forms such that $\rho_{\lambda}^{\text {s.s. }} \approx$ $\sigma_{f_{1}, \lambda} \oplus\left(\sigma_{f_{2}, \lambda} \otimes \chi\right)$ for every $\lambda$.

(ii) There exists $\left\{\varphi_{\lambda}\right\}$ a family of one or two dimensional Galois representations of the absolute Galois group of a number field $E$ that do not extend to $G_{\mathbb{Q}}$ such that $\rho_{\lambda}=\operatorname{Ind} d_{E}^{\mathbb{Q}}\left(\varphi_{\lambda}\right)$ for every $\lambda$.

(iii) Image $\left(\rho_{\lambda}\right)$ is as large as possible for almost every $\lambda$.

In other words, we have generically large image for four-dimensional geometric families of Galois representations satisfying Condition (A), which are non-selfdual, except if the family is reducible or induced from a family of smaller dimension (whose members are not restrictions of representations of $G_{\mathbb{Q}}$ ). For the precise definition of image "as large as possible" see the next section and in particular the statement of Theorem 2.1.

The result can be improved if we restrict to the case of geometric semistable families of representations. Let $N$ be (as before) the product of the primes of bad reduction of the variety $X$ whose cohomology is the source of the Galois representations $\rho_{\lambda}$. By semistable we mean that for every prime $q$ in the ramification set, i.e., $q$ dividing $N$, and for every $\lambda$ relatively prime to $q$, the image of the inertia group at $q$ for the representation $\rho_{\lambda}$ is pro-unipotent. In the semistable case, the result can be proved without imposing Condition (A), and only two possibilities remain, namely, we have the following corollary:

Corollary 1.1. Let $\left\{\rho_{\lambda}\right\}$ be a geometric four-dimensional compatible family of semistable Galois representations of $G_{\mathbb{Q}}$ with coefficients generating an imaginary quadratic field $K$, with Hodge-Tate numbers $\{0,1,2,3\}$ and determinant $\chi^{6}$. Assume that there exists a good reduction prime $p$ such that $\mathbb{Q}\left(a_{p}^{2}\right)=K$ and $b_{p} \neq 0$.

Then one of the following holds:

(i) There exists $f_{1}$ and $f_{2}$ classical cuspidal modular forms with trivial nebentypus such that $\rho_{\lambda}^{\text {s.s. }} \approx \sigma_{f_{1}, \lambda} \oplus\left(\sigma_{f_{2}, \lambda} \otimes \chi\right)$ for every $\lambda$.

(ii) Image $\left(\rho_{\lambda}\right)$ is as large as possible for almost every $\lambda$.

According to the Langlands conjectures, certain automorphic representations of $\mathrm{GL}_{n}$ should always have geometric compatible families of Galois representations attached, meaning that the $L$-function of the automorphic form should agree with the $L$-function of the family of Galois representations. It is also expected that the conductor of the family should agree with the level of the automorphic form. Thus, let 
us assume that we have a compatible family of four-dimensional geometric Galois representations as in the previous theorem (recall that in the context of this paper, geometric implies pure) with the extra assumption that it comes from an algebraic automorphic form $f$ of $\mathrm{GL}_{4}$ over $\mathbb{Q}$. We obtain the following corollary:

Corollary 1.2. Let $\left\{\rho_{f, \lambda}\right\}$ be a geometric compatible family of four-dimensional Galois representations satisfying all the assumptions of Theorem 1.1, and assume that this family is attached to an automorphic form $f$ of $\mathrm{GL}_{4}$ of $\mathbb{Q}$. Then the image is as large as possible for almost every $\lambda$, except if one of the following holds:

(i) $f$ is a weak endoscopic lift

(ii) $f$ is automorphically induced from an automorphic form $g$ of $\mathrm{GL}_{2}$ of a quadratic number field or from a Hecke character of a number field (in both cases, that are not base changed from $\mathbb{Q}$ ).

Remark. Case (i), which corresponds to Case (i) in Theorem 1.1, cannot occur if we assume that the form $f$ is cuspidal.

\section{Previous results and tools}

In our previous paper [6] we studied the images of compatible families of fourdimensional Galois representations and we proved that they are generically large, assuming that certain explicit conditions are satisfied. Since the proofs of the present theorems rely heavily on the analysis and results of [6], we recall its main result (cf. Theorem 4 loc cit).

Theorem 2.1. Let $\left\{\rho_{\lambda}\right\}$ be a compatible family of geometric pure four-dimensional Galois representations with Hodge-Tate weights $\{0,1,2,3\}$ and $N$ the product of the primes in the ramification set, with coefficients in an imaginary quadratic field $K$ and determinant $\chi^{6}$. Assume that the following conditions are satisfied:

Condition (2): There exists a prime $p, p \nmid N$, such that none of the roots of $Q_{p}(x)$, the characteristic polynomial of $\wedge^{2}\left(\rho_{\lambda}\right)$ (Frob $\left.p\right)$, is a number of the form $\eta p^{i}$, where $\eta$ denotes an arbitrary root of unity and $i \in\{1,2,3,4,5\}$.

Condition (3): For every quadratic character $\psi$ unramified outside $N$ there exists a prime $p \nmid N$ with $\psi(p)=-1$ and $p^{3}\left(a_{p}^{2}+\bar{a}_{p}^{2}\right) \neq a_{p} \bar{a}_{p} b_{p}$.

Condition (4): For every cubic character $\phi$ unramified outside $N$ there exists a prime $p \nmid N$ with $\phi(p) \neq 1$ and $a_{p}^{2} b_{p}+p^{6} \neq p^{3} \bar{a}_{p} a_{p}$.

Condition (5): For every quadratic character $\mu$ unramified outside $N$ there exists a prime $p \nmid N$ with $\mu(p)=-1$ and $a_{p} \neq 0$.

Condition (6): There exists a prime $p \nmid N$ such that: $a_{p} \neq \pm \bar{a}_{p}$.

Condition (7): There exists a prime $p \nmid N$ such that $b_{p} \neq 0$ and $a_{p}^{2}$ generates $K$.

Then the image of $\rho_{\lambda}$ is "as large as possible" for almost every prime, i.e., the image of its projectivization $P\left(\rho_{\lambda}\right)$ satisfies:

$$
\operatorname{Image}\left(P\left(\rho_{\lambda}\right)\right) \supseteq \operatorname{PSL}\left(4, \mathbb{Z}_{\ell}\right)
$$

if $\ell$ splits in $K$, and

$$
\operatorname{Image}\left(P\left(\rho_{\lambda}\right)\right)=\operatorname{PSU}\left(4, \mathbb{Z}_{\ell}\right)
$$

if $\ell$ is inert in $K$. 


\section{Remarks.}

1. Observe that Condition (7) is stronger than Condition (6), so in fact only five conditions are required, Conditions (2)-(7). By the way, conditions are numbered starting at (2) since at the beginning of the previous paper there was a "Condition (1)" but it was shown that for the compatible families we are dealing with that condition was always satisfied.

2. The theorem is stated in loc. cit. only for the case of coefficients in a specific imaginary quadratic field because the only available geometric example has coefficients in it, but the proof given applies to the case of coefficients in any imaginary quadratic field.

Another main tool that we have used at several points of our arguments is the information on the action of the inertia at $\ell$. As a consequence of results of FontaineMessing and of Fontaine-Laffaille (cf. [8, 7]) we know that the exponents of the fundamental characters giving the action of the residual representation $\bar{\rho}_{\lambda}$ on the inertia group $I_{\ell}$ must agree with the Hodge-Tate numbers of $\rho_{\lambda}$, more precisely we have (see $[6])$ :

Theorem 2.2. Consider a four-dimensional crystalline representation $\rho_{\lambda}$ with HodgeTate numbers $\{0,1,2,3\}$. Then, if $\ell>3$, the exponents of the fundamental characters giving the action of the (tame) inertia subgroup $I_{\ell}$ of the residual representation $\bar{\rho}_{\lambda}$ are also $\{0,1,2,3\}$. Let $P_{I}:=\mathbb{P}\left(\left.\bar{\rho}_{\lambda}\right|_{I_{\ell}} ^{\text {s.s. }}\right)$ be the projectivization of the image of $I_{\ell}$. Then $P_{I}$ is a cyclic group with order greater than or equal to $\ell-1$.

\section{Proof of the Main Theorem}

Let us start the proof of Theorem 1.1. It follows from Theorem 2.1 that with the running assumptions either the image is as large as possible for almost every prime or the residual representation falls infinitely often in one of the following two cases:

(a) reducible with two two-dimensional irreducible components

(b) imprimitive irreducible.

In fact, Conditions (6) and (7) in Theorem 2.1 hold by assumption and Conditions (2)-(5) in Theorem 2.1 were conditions designed ad hoc to guarantee that Cases (a) and (b) only occur finitely many times: Condition (2) took care of the reducible case with two two-dimensional components (see Section 5.1.2 of [6]) and Conditions (3)-(5) took care of the imprimitive irreducible cases (see Section 5.2 of loc. cit.). Compared to Theorem 2.1, the difference is that now we are not imposing Conditions (2)-(5), and this is why we are allowing these two cases to occur infinitely often.

What remains thus is to characterize intrinsically those geometric families such that Case (a) or (b) occurs for infinitely many primes $\ell$, so as to see that they correspond exactly with items (i) and (ii) in the statement of Theorem 1.1.

3.1. Case (a). Let us suppose that the residual image falls in this case for infinitely many primes $\lambda$. The determinants of the two-dimensional components are $\varepsilon \chi^{i}$ and $\varepsilon^{-1} \chi^{j}$, respectively, where $\varepsilon$ is a character of conductor dividing $d$.

Let us first assume that $i \neq j$, that means $i=1$ and $j=5$ or $i=2$ and $j=4$, consider $\varphi_{\lambda}:=\wedge^{2} \rho_{\lambda}^{s s}$. The characteristic polynomial of $\varphi_{\lambda}\left(\right.$ Frob $\left._{p}\right)$ modulo $\lambda$ has $\varepsilon p^{i}$ and $\varepsilon^{-1} p^{j}$ as roots, for $p \nmid \ell N$. If infinitely many primes $\lambda$ fall in this case, by applying 
Dirichlet's principle (and taking a suitable infinite subset of the set of primes that fall in this case) we can assume that the exponents $i$ and $j$ and the character $\varepsilon$ are independent of $\lambda$. Here we are abusing notation since we are thinking $\varepsilon$ as a character with values on $\mathbb{C}^{*}$, and we use the fact that there are finitely many characters with conductor dividing $d$. We conclude that also in characteristic zero $\varepsilon p^{i}$ and $\varepsilon^{-1} p^{j}$ are roots of the characteristic polynomial of $\varphi_{\lambda}\left(\operatorname{Frob}_{p}\right)$, which is a contradiction with purity. In fact, the roots of the characteristic polynomial of $\varphi_{\lambda}\left(\operatorname{Frob}_{p}\right)=\wedge^{2} \rho_{\lambda}\left(\operatorname{Frob}_{p}\right)$ have absolute value $p^{3}$, as follows from the fact that the determinant of $\rho_{\lambda}\left(\mathrm{Frob}_{p}\right)$ is $p^{6}, \rho_{\lambda}$ is four-dimensional and pure, thus has roots of absolute value $p^{3 / 2}$, and the roots of $\varphi_{\lambda}\left(\right.$ Frob $\left._{p}\right)$ are products of two roots of $\rho_{\lambda}\left(\right.$ Frob $\left._{p}\right)$.

So it is enough to consider the case $i=j$, that is $i=j=3$. In this case by Theorem 2.2 we have that the two two-dimensional irreducible components of the residual $\bmod \lambda$ representation when restricted to the inertia group at $\ell$ are as follows:

$$
\left(\begin{array}{cc}
\chi^{3} & * \\
0 & 1
\end{array}\right)
$$

or

$$
\begin{aligned}
& \left(\begin{array}{cc}
\psi_{2}^{3} & 0 \\
0 & \psi_{2}^{3 \ell}
\end{array}\right), \\
& \left(\begin{array}{cc}
\chi & * \\
0 & 1
\end{array}\right)
\end{aligned}
$$

or

$$
\left(\begin{array}{cc}
\psi_{2} & 0 \\
0 & \psi_{2}^{\ell}
\end{array}\right),
$$

respectively, where $\psi_{2}$ denotes the fundamental character of Level 2.

The determinant of one of these components is $\varepsilon \chi^{3}$. Suppose that for infinitely many of these primes $\lambda$ the corresponding character $\varepsilon$ is odd, i.e., it satisfies $\varepsilon(c)=-1$ where $c$ denotes complex conjugation. Then, for every prime $p$ congruent to -1 modulo $d$ we have that $\varepsilon(p) p^{3}=-p^{3}$ is, modulo infinitely many primes $\lambda$, a root of the characteristic polynomial $Q_{p}(x)$ of $\varphi_{\ell}\left(\right.$ Frob $\left._{p}\right)$. We conclude from this that the same holds in characteristic 0 , i.e., that $-p^{3}$ is a root of the polynomial $Q_{p}(x)$ for every $p$ congruent to -1 modulo $d$, and this contradicts Condition (A).

Thus, we can assume without loss of generality that the character $\varepsilon$ is even, and therefore that the determinants of the two two-dimensional irreducible components are odd.

By Serre's conjecture, which is now a theorem (cf. $[4,10,11])$, this implies that for every $\lambda$ that falls in this case there are two classical cuspidal Hecke eigenforms $f_{1}$ and $f_{2}$ such that

$$
\rho_{\lambda}^{s s} \equiv \sigma_{f_{1}, \lambda} \oplus\left(\sigma_{f_{2}, \lambda} \otimes \chi\right) \quad(\bmod \lambda)
$$

Moreover, applying the strong version of Serre's conjecture (cf. [15]) we can say a lot about the level and weight of these two modular forms: using the description of the action of the inertia group at $\ell$ for the two two-dimensional components that we gave above we deduce that they can be taken of weights 4 and 2 , respectively. Using also the multiplicative property of conductors we conclude that $f_{1} \in S_{4}\left(\Gamma_{1}\left(c_{1}\right)\right)$ and $f_{2} \in S_{2}\left(\Gamma_{1}\left(c_{2}\right)\right)$ with $c_{1} c_{2} \mid c$. As a consequence, we have finitely many possibilities 
for the modular forms $f_{1}$ and $f_{2}$. So, if this occurs for infinitely many primes $\lambda$, by Dirichlet principle we can assume that $f_{1}$ and $f_{2}$ are independent of $\lambda$. Then

$$
\rho_{\lambda}^{s s} \equiv \sigma_{f_{1}, \lambda} \oplus\left(\sigma_{f_{2}, \lambda} \otimes \chi\right) \quad(\bmod \lambda)
$$

for infinitely many $\lambda$. But this implies that the characteristic polynomials at unramified places are the same, since they agree residually in infinitely many characteristics. Let us explain this step in more detail: take a density 0 set of primes $\lambda$ as a subset of the infinite set of reducible primes, and then compare the characteristic polynomials at Frobenius elements corresponding to a density 1 set of primes disjoint from it, thus we conclude that the traces agree on a density 1 set of Frobenius elements, which by Cebotarev is enough to guarantee that the Galois representations are isomorphic, up to semi-simplification. We conclude that:

$$
\rho_{\lambda}^{s s}=\sigma_{f_{1}, \lambda} \oplus\left(\sigma_{f_{2}, \lambda} \otimes \chi\right),
$$

and thus that we are in Case (i) of the theorem.

3.2. Case (b). Assume that the image of the projective residual representation is imprimitive and irreducible. At this point we divide in two cases: the residual representation can be induced from a character or from a two-dimensional representation. Let us consider the case of a character first, i.e., let us assume that for infinitely many primes $\lambda$

$$
\rho_{\lambda} \equiv \operatorname{Ind}_{E}^{\mathbb{Q}}(\psi) \quad(\bmod \lambda),
$$

for some $E$ number field of degree 4 such that its Galois closure has a Galois group sitting in $S_{4}$, and $\psi$ a character of the absolute Galois group $G_{E}$ that cannot be extended to a character of $G_{\mathbb{Q}}$. The character $\psi$ takes values in $\overline{\mathbb{F}}_{\ell}$.

A priory $E$ and $\psi$ depend on the prime $\lambda$, but by Dirichlet principle we can assume without loss of generality that $E$ is independent of $\lambda$ if we know that $E$ does not ramify at $\ell$. Let us show that this is the case, at least for $\ell$ sufficiently large. Moreover, if $F$ is the Galois closure of $E$, let us prove that $F$ must be unramified at $\ell$, for $\ell$ sufficiently large.

We will use the description of the image of inertia at $\ell$ given in Theorem 2.2, valid for primes $\ell>3, \ell \nmid N$. In what follows we will assume that $\ell \nmid N$ and that $\ell>5$. If we consider the image of the projectivization $\mathbb{P}\left(\bar{\rho}_{\lambda}\right)$, then in this imprimitive irreducible case this image is a group $H$ that fits in an exact sequence:

$$
0 \rightarrow C \rightarrow H \rightarrow S \rightarrow 0
$$

where $C$ is cyclic and $S$ is certain non-trivial subgroup of $S_{4}$. In particular $|S| \leq 24$. Note that the quotient $S$ of $H$ is the Galois group $\operatorname{Gal}(F / \mathbb{Q})$. The subgroup $C$ is the projectivization of a diagonal subgroup in $\mathrm{GL}_{4}\left(\overline{\mathbb{F}}_{\ell}\right)$ and $H$ is contained in the normalizer of $C$. Moreover, since elements in $H \backslash C$ act on $C$ as permutation matrices, it follows that a matrix in $C$ having four different eigenvalues will not conmute with any element of $H \backslash C$. From this, using the description of the projective image inertia at $\ell$, the fact that its order grows with $\ell$ and that it is a cyclic group, and the fact that the four digits of the exponent of the fundamental character are different, we see that if $\ell$ is sufficiently large this inertia group must be contained in $C$ : if not, for large $\ell$ a large but proper subgroup of this inertia group would be contained in $C$ and the rest in $H \backslash C$, thus it would be non-abelian. Hence, $F$ is unramified at $\ell$. 
We conclude that the ramification set of $E$ is contained in the set of prime divisors of $N$, and the degree of $E$ is also bounded. By Hermite-Minkowski, there are only finitely many such fields $E$, thus we can assume (by taking a suitable infinite subset of the set of primes that fall in this case) that $E$ is independent of $\lambda$. Moreover, we have certain uniformity on the characters $\psi$, even if they are only defined as residual characters, because their conductors (i.e., the prime-to- $\ell$ part of their Artin conductors) must be bounded in terms of the conductor $c$ of the family $\rho_{\lambda}$ (this is automatic, use for example the formula giving the behavior of conductors under base change) and there are finitely many possibilities for the exponents of the fundamental characters describing the ramification at the prime $\ell$ because of Theorem 2.2. In this situation, we can apply a result of Serre (see [13], Section 3, Theorem 1, for the case of coefficients in $\mathbb{Z}$ and [12], Theorem (MT 2), for the generalization to the general case) to the restriction to the Galois closure of $E$ of the given family of Galois representations, and conclude that residual reducibility for infinitely many primes gives reducibility of the $\lambda$-adic representations, for every $\lambda$, thus:

$$
\rho_{\lambda}=\operatorname{Ind}_{E}^{\mathbb{Q}}(\phi),
$$

for all $\lambda$, for some character $\phi$ of the absolute Galois group of $E$. This case is included in item (ii) of the theorem.

Finally, we have to consider the second possibility. Assume that for infinitely many $\lambda$, we have

$$
\rho_{\lambda} \equiv \operatorname{Ind}_{E}^{\mathbb{Q}}\left(\sigma_{\lambda}\right) \quad(\bmod \lambda)
$$

where $\sigma_{\lambda}$ is a two-dimensional representation of $G_{E}$ that is not the restriction of a two-dimensional representation of $G_{\mathbb{Q}}$, for $E$ a quadratic extension of $\mathbb{Q}$. As we have shown in the previous case, we see in the same way that for $\ell$ sufficiently large the field $E$ will be unramified at $\ell$, thus $E$ can be assumed (applying the Dirichlet principle) independent of $\lambda$. This induced representation is by assumption irreducible, then

$$
a_{p} \equiv \operatorname{tr}\left(\overline{\rho_{\lambda}}\left(\operatorname{Frob}_{p}\right)\right) \equiv 0 \quad(\bmod \lambda),
$$

for all $p \nmid N \ell$ a prime inert in $E / \mathbb{Q}$. Since this holds for infinitely many primes $\lambda$, we obtain $a_{p}=0$, for a set of primes $p$ of density $1 / 2$ (the primes inert in $E / \mathbb{Q}$ ). By [3], Theorem 3.2, this implies that $\rho_{\lambda}=\operatorname{Ind}_{E}^{\mathbb{Q}}\left(\sigma_{\lambda}^{\prime}\right)$, for some two-dimensional family of $\lambda$-adic representations $\left\{\sigma_{\lambda}^{\prime}\right\}$ of $G_{E}$. This concludes the proof, since this case is also included in item (ii) of the theorem.

\section{Proof of the corollaries}

4.1. Proof of 1.1. To begin the proof of this corollary, let us explain how we can get rid of Condition (A). Recall that in the proof of Theorem 1.1 this condition was used in the case of reducible residual image with two two-dimensional irreducible components to gain control on the Dirichlet character $\varepsilon$ in the determinant of an irreducible component. Let us show that in the semistable case this Dirichlet character has to be trivial, for sufficiently large $\ell$.

The character $\varepsilon$ only ramifies at primes in $N$, and its conductor is bounded by $d$. From this we see that the order of $\varepsilon$ is bounded independently of $\lambda$. By the semistability assumption, the ramification at any prime dividing $N$ of any residual 
$\bmod \lambda$ representation in the family is given by an $\ell$-group. We conclude that for sufficiently large $\ell$ the character $\varepsilon$ has to be trivial.

Observe that what we have just proved has two consequences: first, that we do not need Condition (A) to guarantee that in the residually reducible case the two irreducible components will be odd two-dimensional representations; and second, that when proving modularity of these two two-dimensional components as in the proof of Theorem 1.1 since the character $\varepsilon$ is now trivial the two modular forms $f_{1}$ and $f_{2}$ have trivial nebentypus.

Finally, let us explain why in the semistable case the family of representations cannot be induced from a family of smaller dimension. As we saw in the proof of Theorem 1.1, this case occurs if and only if the residual representations are induced for almost every $\lambda$. Thus, if the family were induced, part of the ramification of the residual mod $\lambda$ representations at the primes in $N$ will come, at least for almost every $\lambda$, from the induction. Namely, there will be a extension of $\mathbb{Q}$, either quadratic or quartic such that its Galois closure has Galois group a subgroup of $S_{4}$ such that some of the primes $q$ dividing $N$ will ramify in this extension and the ramification of the residual representations at $q$ will thus have a quotient of order 2,3 , or 4 . But the semistability assumption implies that the ramification at $q$ of these residual representation is an $\ell$-group, thus this cannot happen if $\ell>3$.

4.2. Proof of 1.2. This corollary follows easily from Theorem 1.1. In fact, if the four-dimensional compatible family is attached to $f$, we already know that the image fails to be as large as possible for almost every prime only in very specific cases: If the family is as in item (i) of Theorem 1.1 then $f$ is a weak endoscopic lift by definition.

If the family is as in item (ii) of the theorem, either it is induced from a onedimensional or from a two-dimensional family. In the first case, clearly $f$ is automorphically induced from a Hecke character, the character such that the corresponding $\ell$-adic representations appear in the formula in item (ii). In the second case, it remains to show that being the Galois representations induced from a quadratic number field $E$ the form $f$ also must be automorphically induced from $E$.

We saw during the proof of Theorem 1.1 that in such an induced case (we are also assuming irreducibility because reducible cases are covered by item (i)) the family satisfies $a_{p}=0$ for all primes $p$ inert in $E / \mathbb{Q}$. Thus it satisfies:

$$
\rho_{f, \lambda}=\rho_{f, \lambda} \otimes \mu
$$

where $\mu$ is the quadratic character of the extension $E / \mathbb{Q}$. By strong multiplicity one (cf. [9]), this implies: $f=f \otimes \mu$.

Hence, we can apply the characterization of automorphic forms being in the image of solvable base change given in [1], Chapter 3, Theorem 4.2 (b) and Lemma 6.6 and conclude that $f$ is automorphically induced from an automorphic form of $\mathrm{GL}_{2}$ of the quadratic number field $E$. This concludes the proof of the corollary.

\section{Acknowledgments}

Research is partially supported by MICINN grant MTM2009-07024. The first named author is also partially supported by an ICREA Academia Research Prize. 


\section{References}

[1] J. Arthur and L. Clozel, Simple Algebras, base change and the advanced theory of the trace formula, Princeton University Press, 1989.

[2] P. Berthelot, Altérations de variétés algébriques [d'après A. J. de Jong], in 'Séminaire Bourbaki', 1995/96, exp. 815, Astérisque, 1997, 273-311.

[3] C. Consani and J. Scholten, Arithmetic on a quintic threefold, Internat. J. Math. 12 (2001), 943-972.

[4] L. Dieulefait, Remarks on Serre's modularity conjecture, preprint (2006), available at www.arxiv.org

[5] L. Dieulefait and N. Vila, On the images of modular and geometric three-dimensional Galois representations, Amer. J. Math. 126 (2004), 335-361.

[6] L. Dieulefait and N. Vila, Geometric families of 4-dimensional Galois representations with generically large images, Math. Z. 259 (2008), 897-893.

[7] J.M. Fontaine and G. Laffaille, Construction de représentations p-adiques, Ann. Scient. Éc. Norm. Sup., $4^{e}$ série, t. 15 (1982), 547-608.

[8] J.M. Fontaine and W. Messing, p-adic periods and p-adic etale cohomology, in 'Currents trends in arithmetical algebraic geometry (Arcata, Calif., 1985)', Contemporary Mathematics 67 (1987), 179-207.

[9] H. Jacquet and J. Shalika, Euler products and the classification of automorphic forms (I, II), Amer. J. Math. 103 (1981), 499-558 and 777-815.

[10] C. Khare and J-P. Wintenberger, Serre's modularity conjecture (I, II), Invent. Math. 178 (2009), 485-504 and 505-586.

[11] M. Kisin, Modularity of 2-adic Barsotti-Tate representations, Invent. Math. 178 (2009), 587-634.

[12] K.A. Ribet, Galois action on division points of abelian varieties with real multiplications, Amer. J. Math. 98 (1976), 751-804.

[13] J.-P. Serre, Propriétés galoisiennes des points d'ordre fini des courbes elliptiques, Invent. Math. 15 (1972), 259-331.

[14] J.-P. Serre, Oeuvres, 4, Springer-Verlag, New York, 2000, 1-55.

[15] J.-P. Serre, Sur les représentations modulaires de degré 2 de Gal( $\overline{\mathbf{Q}} / \mathbf{Q})$, Duke Math. J. 54 (1987), 179-230.

Dept. D’Àlgebra i Geometria, Universitat de Barcelona, Gran Via de les Corts CataLANES 585, 08007 - BARCELONA, SPAIN

E-mail address: ldieulefait@ub.edu

E-mail address: nuriavila@ub.edu 